\title{
Episodic retrieval and the SNARC effect
}

\author{
Peter Dixon ${ }^{1}$
}

Published online: 27 February 2017

(C) Psychonomic Society, Inc. 2017

\begin{abstract}
The SNARC effect is the finding that left-hand responses are faster to small digits and right-hand responses are faster to large digits. I tested an episodic-retrieval account of the SNARC effect in which it is assumed that the response time varies as a function of the prior trial episodes available in working memory. Blocks of trials were constructed in which two digits were repeated $75 \%$ of the time; presumably, these two "focus" digits would be readily available in working memory. Under such circumstances, there was no overall SNARC effect. Instead, response times were faster when the stimulus-response mapping was consistent with most of the trials in the block. This means that the usual SNARC effect was obtained when the focus digits were consistent with that effect, and that a reverse SNARC effect was obtained when responses to those digits were inconsistent with it.
\end{abstract}

Keywords Stimulus-response compatibility $\cdot$ Episodic memory $\cdot$ Working memory

The SNARC (spatial-numerical association of response codes) effect is a stimulus-response compatibility effect in which left-hand responses are faster when the stimulus is a small digit and right-hand responses are faster when the stimulus is a large digit. An important aspect of this effect is that it occurs even in tasks such as parity judgments, in which digit magnitude is unrelated to the required response. Dehaene, Bossini, and Giraux (1993) originally explained the effect by assuming that digits automatically activated the mental representation of a number line, with small digits on the left and

Peter Dixon

peter.dixon@ualberta.ca

1 University of Alberta, Edmonton, Alberta, Canada large digits on the right. Thus, faster responses occur when "left" or "right" spatial codes match the left or right manual response. However, van Dijck and Fias (2011) proposed an alternative account in which a digit stimulus cues the retrieval of previous stimuli from working memory. According to this account, the SNARC effect arises because of the use of a spatial representation of those prior stimuli. The present research provides evidence for a version of this working memory account by manipulating the contents of working memory across trials.

Several lines of evidence support the ideas that spatial representations are associated with number magnitude and that these could provide a basis for the SNARC effect. For example, numbers appear to spontaneously cue shifts of spatial attention (Fischer, Castel, Dodd, \& Pratt, 2003); hemineglect patients have distortions when asked to bisect a number line (Zorzi, Priftis, \& Umiltà, 2002); and spatial working memory load eliminates the SNARC effect in magnitude comparison tasks (Herrera, Macizo, \& Semenza, 2008). Spatial representations might affect response times in several ways. In keeping with Dehaene et al.'s (1993) original conception of a mental number line, digits may be mapped onto positions in space from left to right (cf. Ishihara et al., 2006), and responses can be slower when they correspond to different positions or effectors. Thus, a small digit would be mapped onto a position on the left and would be inconsistent with a right-hand response or a movement to the right in space. Alternatively, the spatial representations may be more abstract. For example, Gevers, Verguts, Reynvoet, Fias, and Caessens (2006) proposed a model in which "left" and "right" spatial codes are automatically activated by the presentation of small and large digits, and these codes compete with the activation of "left" and "right" codes for the response.

Although this evidence indicates that spatial representations are likely to be in involved in the SNARC effect, other 
results suggests that the effect is labile and can be affected by a variety of manipulations. For example, shifts of spatial attention may depend on the strategies that subjects use, rather than occurring automatically (Fattorini, Pinto, Rotondaro, \& Doricchi, 2015). Similarly, priming subjects to think of digits in terms of their position on a clock face (in which case larger digits are on the left rather than the right) reverses the effect (Bächtold, Baumüller, \& Brugger, 1998). The effect is also "range-dependent," so that, for example, responses are faster for left-hand responses to stimuli that are small relative to others in the stimulus set (Dehaene et al., 1993). For bilingual subjects, the SNARC effect can be eliminated by reading a single word in Hebrew (in which the effect is not usually obtained) and reinstated by reading a word in Russian (Fischer, Shaki, \& Cruise, 2009). Similarly, the magnitude of the SNARC effect is affected by reading recipes in which digits appear in SNARC-compatible or -incompatible positions (Fischer, Mills, \& Shaki, 2010). Gevers et al. (2010) demonstrated circumstances in which the effect is mediated by verbal rather than spatial representations. As another example, Tan and Dixon (2011) found no SNARC effect when the digit was the same as the one presented on the previous trial. Thus, it seems clear that the simple idea that small digits are mapped to "left" and large digits to "right" is inadequate, and that more complex mechanisms or more flexible representations must be involved.

One candidate for a more nuanced account was provided by van Dijck and Fias (2011), who argued that the contents of working memory were important. In their experiments, subjects were given sequences of digits to maintain in order in working memory. Van Dijck and Fias observed that responses were faster when left-hand responses were made to digits early in the sequence, and right-hand responses were faster to digits later in the sequence. They hypothesized that the digit load was maintained spatially from left to right, producing the observed compatibility effect, and suggested that when subjects do not have to maintain a digit load, the digits used on prior trial episodes may be available in working memory and organized spatially.

However, previous research on stimulus-response repetition effects has set several constraints on the role of prior trial episodes. M. C. Smith (1968), for example, demonstrated faster responses when both the stimulus and the response repeated from the previous trial (see also Bertelson, 1965; Rabbitt, 1968). Pashler and Baylis (1991) proposed an account of such repetition effects in which a transient link was generated between the stimulus and the response, allowing response selection to be short circuited. A similar idea was described by E. E. Smith, Chase, and Smith (1973), in which prior stimulus-response mappings were maintained in shortterm memory and used to make rapid responses. Critically, however, the repetition effects based on prior trial episodes seem to be limited to repetition of either the preceding stimulus or the stimulus before that (Pashler \& Baylis, 1991; see also E. E. Smith et al., 1973). Although these mechanisms involve working memory, the limitation of repetition effects to the preceding trial or two casts doubt on the idea that subjects would spontaneously maintain an extended list of prior stimuli, as was suggested by van Dijck and Fias (2011).

In the present research, I evaluated whether a SNARC effect could be due to the retrieval of stimulus-response pairs, without assuming extensive memory of prior trials. In particular, I manipulated the structure of trial blocks in order to vary the nature of the prior episodes that would be readily available in working memory. In each block of trials, an odd digit and an even digit were selected as "focus" stimuli; these focus items were presented on $75 \%$ of the trials in that block. Thus, one could be relatively certain that episodes with these two stimuli would likely be salient and available in working memory. Because of this availability, I expected that responses to the focus digits would be fast. Indeed, many of these trials would involve stimulus-response repetitions. Across blocks of trials, the focus digits were selected to be either SNARC compatible (i.e., a large digit and a right-hand response or a small digit and a left-hand response) or SNARC incompatible. The question of interest was whether the nature of the focus digits would also affect responses to the rest of the digits (i.e., the nonfocus digits). The nonfocus digits could also be SNARC compatible or incompatible, but, in addition, they could appear in blocks in which the focus digits were either SNARC compatible or SNARC incompatible. Across blocks, then, the nonfocus digits were equally often SNARC compatible and incompatible, and appeared equally often in the context of SNARCcompatible or -incompatible focus digits.

\section{Method}

On each trial, subjects were presented with a digit and were asked to decide whether the digit was odd or even. Responses were made by pressing the " $D$ " or the " $L$ " key with subjects" left or right index finger. In each block of 24 trials, two possible focus digits, one odd and one even, appeared on $75 \%$ of the trials.

\section{Procedure}

Each block began with a message indicating the stimulusresponse mapping for that block, which remained on the screen for a minimum of $3 \mathrm{~s}$. Subjects then initiated the block by pressing the space bar. On each trial, the stimulus digit was displayed in the center of the screen until the subject responded by pressing the " $\mathrm{D}$ " or the "L" key. If the response was correct, the stimulus for the next trial was presented after $1 \mathrm{~s}$. If the response was incorrect, the message "Incorrect" was presented for $2 \mathrm{~s}$, followed by the 1-s delay before the next 
trial. Stimuli were presented on a $51-\mathrm{cm}$ iMac screen with a resolution of $98 \mathrm{dpi}$ at an approximate distance of $50 \mathrm{~cm}$, in 24-point Helvetica font. At that distance, the stimuli subtended approximately $0.5^{\circ}$ of visual angle.

\section{Design}

The stimuli consisted of the digits $1-4$ and 6-9. Trials were arranged in 32 blocks of 24 . The stimulus-response mapping of odd-even to left-right alternated every block. In each block, two focus digits were selected. One focus digit was odd, and the other was even. In addition, one focus digit was large (greater than 5), and one was small (smaller than 5). Thus, a total of eight pairs of focus digits were possible. Across blocks, each of those eight pairs was used equally often in blocks in which the mapping for the focus digits was SNARC compatible or incompatible. This is illustrated in Table 1, in which there is a row for each of the eight possible pairs of focus digits and a column for each of the two stimulus-response mappings. The entries in the table indicate whether a block using that pair of focus digits provides a SNARC-compatible or -incompatible context. Within a block, each focus digit was used nine times, and the remaining six (nonfocus) digits were each used once. Thus, across blocks, the nonfocus digits were either SNARC compatible or SNARC incompatible and either focus compatible or focus incompatible.

\section{Subjects}

The subjects were 21 undergraduates at the University of Alberta who were given an honorarium of $\$ 15$ for their participation. Ten of the subjects began with the odd-right/evenleft mapping, and 11 began with the reverse mapping.

Table 1 Focus digit compatibility as a function of stimulus-response mapping

\begin{tabular}{lll}
\hline $\begin{array}{l}\text { Possible Focus Digit } \\
\text { Pairs }\end{array}$ & \multicolumn{2}{l}{ Stimulus Response Mapping } \\
\cline { 2 - 3 } & $\begin{array}{l}\text { Odd-Left/Even- } \\
\text { Right }\end{array}$ & $\begin{array}{l}\text { Odd-Right/Even- } \\
\text { Left }\end{array}$ \\
\hline 1,6 & Compatible & Incompatible \\
1,8 & Compatible & Incompatible \\
2,7 & Incompatible & Compatible \\
2,9 & Incompatible & Compatible \\
3,6 & Compatible & Incompatible \\
3,8 & Compatible & Incompatible \\
4,7 & Incompatible & Compatible \\
4,9 & Incompatible & Compatible \\
\hline
\end{tabular}

\section{Analysis}

The median correct response time was calculated for each subject, stimulus type (focus or nonfocus), SNARC compatibility, and compatibility of the focus digits. The responses to focus and to nonfocus digits were analyzed separately. Evidence for different patterns of results was assessed by comparing nested linear mixed-effects models. In all of the models compared, the mean response time was assumed to vary across subjects, and the models differed only in the fixed effects that were included. More complex random-effects structures did not improve the models. The models were fit using the program lmer (Bates, Maechler, Bolker, \& Walker, 2013) running in the $\mathrm{R}$ statistical environment ( $\mathrm{R}$ Core Team, 2014). The models were compared by computing the maximum likelihood ratio and then adjusting for the differing degrees of freedom by using the Akaike information criterion (AIC; Akaike, 1973; cf. Glover \& Dixon, 2004). We use the symbol $\lambda_{\text {adj }}$ to indicate such adjusted likelihood ratios. Burnham and Anderson (2002) refer to these statistics as evidence ratios. Ratios very much larger than 1 indicate that one model is clearly superior to the other model, whereas ratios close to 1 indicate weak or little evidence that one model is better. By way of comparison, in some prototypical hypothesis-testing situations, an adjusted likelihood ratio of about 3 corresponds to an attained $p$ value of .05. Comparing models in this way is tantamount to selecting models on the basis of AIC values, a common model selection technique. Response accuracy was analyzed similarly, except that generalized linear mixedeffects models were fit using the binomial family and a logistic link function (cf. Dixon, 2008) with the R program glmer (Bates et al., 2013).

\section{Results}

As is shown in Fig. 1, there was a substantial interaction between the SNARC compatibility effect and the nature of the focus digit context for the nonfocus digits. The nature of this interaction is that when the focus digits were SNARC compatible, the nonfocus digits seemed to show the usual SNARC effect, with faster responses for compatible and slower responses for incompatible stimulus-response mappings. However, when the focus digits were SNARC incompatible, the effect for the nonfocus digits appeared to reverse, with faster responses for incompatible mappings. There seemed to be no difference overall between SNARC-compatible and SNARC-incompatible trials, nor an appreciable overall effect of the nature of the focus digit context. This interpretation was supported by a comparison of nested linear models. Comparing a model with an overall effect of SNARC compatibility to a null model indicated little evidence for the compatibility effect $\left(\lambda_{\text {adj }}=0.37\right)$. Similarly, adding the effect of 


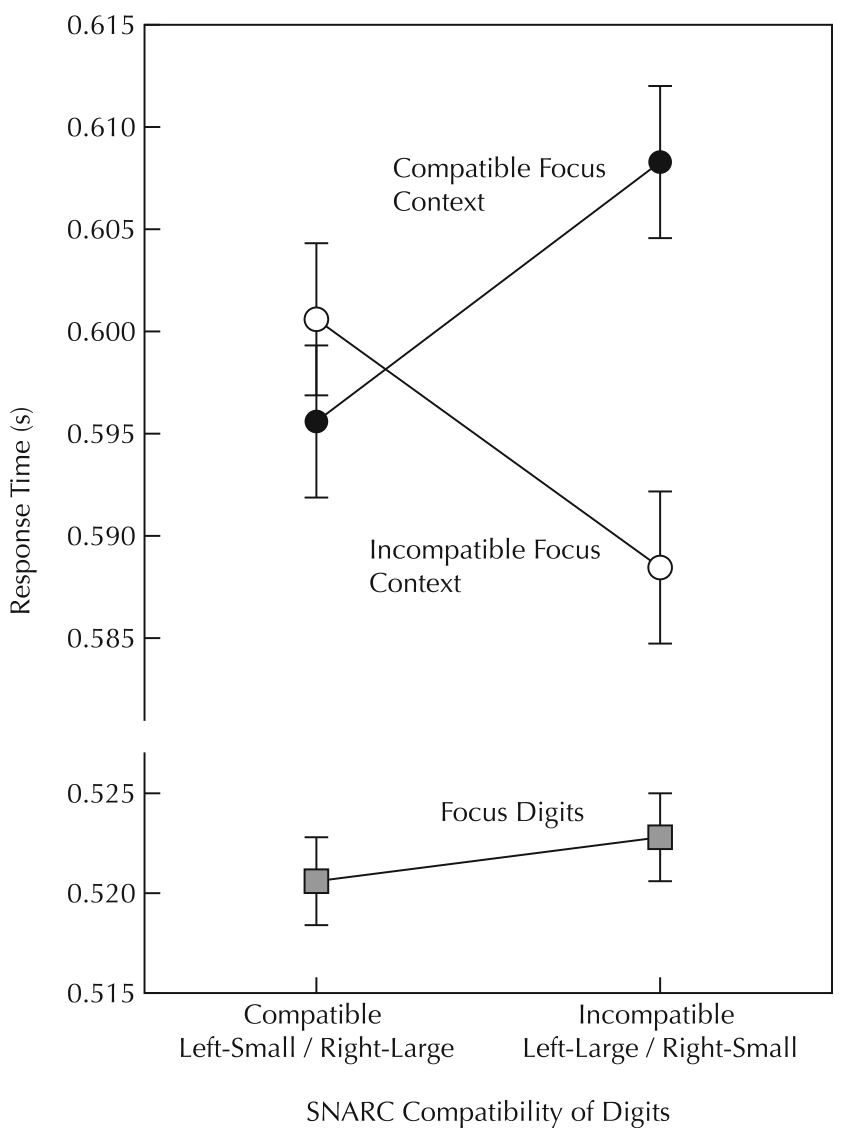

Fig. 1 Median response times as a function of stimulus and block type. Error bars were computed from the standard errors of the estimated effect sizes in a full-model fit, excluding the overall mean

context failed to improve the model very much $\left(\lambda_{\text {adj }}=1.33\right)$. However, a model with an interaction between the two factors was substantially better than the additive model $\left(\lambda_{\text {adj }}=18.58\right)$.

The lower panel in Fig. 1 shows the effect of compatibility on the focus digits. As expected, these response times were substantially faster than those for nonfocus digits. There was little evidence of a SNARC effect: A model with the compatibility effect was no better than the null model $\left(\lambda_{\text {adj }}=0.42\right)$. This result is consistent with previous research demonstrating a negligible SNARC effect when the stimulus is the same as that on the previous trial (Tan \& Dixon, 2011).

Similar results were found for accuracy (shown in Table 2). In particular, for nonfocus digits there was substantial

Table 2 Proportions correct (and standard errors)

\begin{tabular}{lll}
\hline Stimulus Type & \multicolumn{2}{l}{ Focus Context } \\
\cline { 2 - 3 } & Compatible & Incompatible \\
\hline Compatible nonfocus & $.961(.005)$ & $.942(.006)$ \\
Incompatible nonfocus & $.930(.007)$ & $.960(.006)$ \\
Compatible focus & $.978(.001)$ & \\
Incompatible focus & & $.978(.001)$ \\
\hline
\end{tabular}

evidence for a model that included the interaction of compatibility and context, relative to a purely additive model $\left(\lambda_{\text {adj }}=\right.$ 296.86). For the focus digits, there was no evidence of an effect of compatibility $\left(\lambda_{\text {adj }}=0.37\right)$.

\section{Discussion}

A simple description of the results shown in Fig. 1 is that the responses to the nonfocus digits were faster when the stimulus-response mapping matched that used for the focus digits. Thus, for example, the responses for a large nonfocus digit were fast when the digit required the same response as the large focus digit that was encountered many times in that block. In contrast, no evidence emerged for a SNARC effect, independent of the block context. My hypothesis was that because the focus digit occurred often in a block of trials, episodic-memory traces of the stimulus-response mapping would be available in working memory. These memory traces would allow a rapid response when the stimulus mapping on the current trial was consistent with that in the prior episode.

The results demonstrate that the contents of working memory can affect the pattern of responses to large and small digits. As found by M. C. Smith (1968) and others, exact stimulus repetitions are fast, presumably because of the availability of the associated response in working memory. However, my interpretation is that responses to nonfocus (and nonrepeated) stimuli could also be relatively fast if the current stimulus was similar in terms of magnitude to an available episode that had required the same response. For example, if the current stimulus was large, I assume that it would be similar to prior episodes involving the large focus digit. If that current large stimulus required the same response as the large focus digit, the response would be relatively fast, because it would be consistent with the prior episodes for that focus digit. In contrast, if a large stimulus required a different response than did the large focus digit, there would be some interference from the prior episodes, and the response would be slower. In this way, the stimulus-response mapping on the prior focus-digit trials would affect the response time for the balance of the stimuli. Although this interpretation implicates working memory, it is possible that spatial representations of the digits might be involved. Unlike the results of van Dijck and Fias (2011), there was no taskirrelevant requirement to maintain information in memory: The contents of working memory were manipulated simply by varying the stimulus composition of a block. Thus, the present paradigm is closer to the circumstances in typical demonstrations of the SNARC effect. 
Table 3 Interaction magnitudes (in seconds) as a function of block portion

\begin{tabular}{ll}
\hline Block Portion & Interaction Magnitude (and Standard Error) \\
\hline 1 & $0.022(0.019)$ \\
2 & $0.049(0.019)$ \\
3 & $0.037(0.019)$ \\
\hline
\end{tabular}

An alternative interpretation of the present results is that the interaction between the nature of the focus digits and SNARC compatibility is due to gradual learning of the stimulus probabilities, rather than to the presence of prior trial episodes in working memory. For example, Gevers et al. (2006) proposed an activation-based model of the SNARC effect, and it might be possible to produce the present pattern of results by changing the connection weights on the basis of experience with the stimulus frequency over the course of a block. To assess this type of possibility, each block was divided into thirds, and the magnitude of the focus-compatibility interaction was calculated in each third. If the interaction was related to some form of incremental learning process, the magnitude of the effect should increase across thirds. If, on the other hand, the interaction was due to the contents of working memory, the interaction might be somewhat smaller in the first third of the block (because the focus digits might not yet have been encountered in some cases), but there would be no reason to expect the interaction to increase from the second to the third part of the block. The results are shown in Table 3. Although the interaction increased somewhat from the first to the second part, it was numerically smaller in the third part. This pattern of results is consistent with a working memory account and provides no support for a role of incremental learning.

Although these results contribute to the literature on the malleability of the SNARC effect, by themselves they do not explain why the usual SNARC effect occurs when digits appear equally often in a block. One possibility is that stimulusresponse pairs that are SNARC consistent (i.e., left for a small digit or right for a large digit) are more memorable, and consequently more likely to be retained over trials. Such differences in memorability may occur because of a SNARCconsistent prior association between digits and the verbal codes of "left" and "right" (cf. Dixon \& Westbury, 2012; Hutchinson \& Louwerse, 2014), or because of a tendency to organize the contents of working memory spatially, with smaller digits on the left (van Dijck \& Fias, 2011). Although these ideas are similar to previous accounts of the SNARC effect, the present results suggest that the contents of working memory may be the proximal cause of the response time effects, and that other factors affect response times only indirectly.

\section{References}

Akaike, H. (1973). Information theory and an extension of the maximum likelihood principle. In B. N. Petrov \& F. Csáki (Eds.), Second international symposium on information theory (pp. 267-281). Budapest: Akadémia Kiadó.

Bächtold, D., Baumüller, M., \& Brugger, P. (1998). Stimulus-response compatibility in representational space. Neuropsychologia, 36, 731735.

Bates, D., Maechler, M., Bolker, B., \& Walker, S. (2013). lme4: Linear mixed-effects models using Eigen and S4 (R package version 1.0-5) [Computer Software]. Retrieved from http://CRAN.R-project.org/ package $=1 \mathrm{me} 4$

Bertelson, P. (1965). Serial choice reaction-time as a function of response versus signal-and-response repetition. Nature, 206, 217-218.

Burnham, K. P., \& Anderson, D. R. (2002). Model selection and multimodel inference: A practical information-theoretic approach. New York: Springer.

Dehaene, S., Bossini, S., \& Giraux, P. (1993). The mental representation of parity and number magnitude. Journal of Experimental Psychology: General, 122, 371-396. doi:10.1037/0096-3445.122. 3.371

Dixon, P. (2008). Models of accuracy in repeated-measures designs. Journal of Memory and Language, 59, 447-456. doi:10.1016/j. jml.2007.11.004

Dixon, P., \& Westbury, C. (2012). A basis for the SNARC effect in in language. Poster presented at the meeting of the Psychonomic Society. Minneapolis, MN.

Fattorini, E., Pinto, M., Rotondaro, F., \& Doricchi, F. (2015). Perceiving numbers does not cause automatic shifts of spatial attention. Cortex, 73, 298-316. doi:10.1016/j.cortex.2015.09.007

Fischer, M. H., Castel, A. D., Dodd, M. D., \& Pratt, J. (2003). Perceiving numbers causes spatial shifts of attention. Nature Neuroscience, 6 , 555-556. doi:10.1038/nn1066

Fischer, M. H., Mills, R. A., \& Shaki, S. (2010). How to cook a SNARC: Number placement in text rapidly changes spatial-numerical associations. Brain and Cognition, 72, 333-336. doi:10.1016/j.bandc. 2009.10.010

Fischer, M. H., Shaki, S., \& Cruise, A. (2009). It takes just one word to quash a SNARC. Experimental Psychology, 56, 361-366. doi:10. 1027/1618-3169.56.5.361

Gevers, W., Santens, S., Dhooge, E., Chen, Q., Van den Bossche, L., Fias, W., \& Verguts, T. (2010). Verbal-spatial and visuospatial coding of number-space interactions. Journal of Experimental Psychology: General, 139, 180-190. doi:10.1037/a0017688

Gevers, W., Verguts, T., Reynvoet, B., Fias, W., \& Caessens, B. (2006). Numbers and space: A computational model of the SNARC effect. Journal of Experimental Psychology: Human Perception and Performance, 32, 32-44. doi:10.1037/0096-1523.32.1.32

Glover, S., \& Dixon, P. (2004). Likelihood ratios: A simple and flexible statistic for empirical psychologists. Psychonomic Bulletin \& Review, 11, 791-806. doi:10.3758/BF03196706

Herrera, A., Macizo, P., \& Semenza, C. (2008). The role of working memory in the association between number magnitude and space. Acta Psychologica, 128, 225-237. doi:10.1016/j.actpsy.2008.01. 002

Hutchinson, S., \& Louwerse, M. M. (2014). Language statistics explain the spatial-numerical association of response codes. Psychonomic Bulletin \& Review, 21, 470-478. doi:10.3758/s13423-013-0492-2

Ishihara, M., Jacquin-Courtois, S., Flory, V., Salemme, R., Imanaka, K., \& Rossetti, Y. (2006). Interaction between space and number representations during motor preparation in manual aiming. Neuropsychologia, 44, 1009-1016. doi:10.1016/j.neuropsychologia. 2005.11.008 
Pashler, H., \& Baylis, G. C. (1991). Procedural learning: II. Intertrial repetition effects in speeded-choice tasks. Journal of Experimental Psychology: Learning, Memory, and Cognition, 17, 33-48. doi:10. 1037/0278-7393.17.1.33

R Core Team. (2014). R: A language and environment for statistical computing. Vienna: R Foundation for Statistical Computing. Retrieved from www.R-project.org/

Rabbitt, P. M. (1968). Repetition effects and signal classification strategies in serial choice-response tasks. Quarterly Journal of Experimental Psychology, 20, 232-240. doi:10.1080/ 14640746808400157

Smith, M. C. (1968). Repetition effect and short-term memory. Journal of Experimental Psychology, 77, 435-439. doi:10.1037/h0021293
Smith, E. E., Chase, W. G., \& Smith, P. G. (1973). Stimulus and response repetition effects in retrieval from short-term memory: Trace decay and memory search. Journal of Experimental Psychology, 98, 413422. doi: $10.1037 / \mathrm{h} 0034398$

Tan, S., \& Dixon, P. (2011). Repetition and the SNARC effect with oneand two-digit numbers. Canadian Journal of Experimental Psychology, 65, 84-97. doi:10.1037/a0022368

van Dijck, J. P., \& Fias, W. (2011). A working memory account for spatial-numerical associations. Cognition, 119, 114-119. doi:10. 1016/j.cognition.2010.12.013

Zorzi, M., Priftis, K., \& Umiltà, C. (2002). Brain damage: Neglect disrupts the mental number line. Nature, 417, 138-139. doi:10.1038/ 417138a 